\title{
revistainvi
}

Volumen 36, nro. 102, agosto 2021 ISSN 0718-1299

\section{¿Al lado del camino? Inventariando estrategias de autogestión del hábitat en Chile}

Recibido: 2020-04-28

Aceptado: 2021-04-01

Cómo citar este artículo:

Moreno Crossley, J. C. (2021). ¿Al lado del camino? Inventariando estrategias de autogestión del hábitat en Chile. Revista INVI, 36(102), 279-301. https://doi.org/10.4067/S0718-83582021000200279

\section{Juan Cristóbal Moreno Crossley}

Estudiante de Doctorado Universidad de Chile, Santiago, Chile,jcristobalmoreno@gmail.com https://orcid.org/0000-0003-1542-8248 


\section{¿Al lado del camino? \\ Inventariando estrategias \\ de autogestión del hábitat en Chile}

Palabras clave: autogestión; hábitat; vivienda; políticas públicas; Chile.

\section{Resumen}

Reconociendo su carácter múltiple y rechazando su mal adjudicada calificación de prácticas residuales o periféricas, el presente artículo aborda la tarea de describir, catalogar y cuantificar algunas de las principales estrategias contemporáneas de autogestión del hábitat en Chile, subrayando cómo en ellas pueden identificarse claves significativas para promover mejoras en las condiciones de vida de algunos grupos de población. Para esto se aplica una metodología cuantitativa y descriptiva, basada en el análisis de los datos de Encuesta Casen 2017, que busca estimar la incidencia de diferentes prácticas ligadas a la autogestión del hábitat entre los hogares del primer quintil de ingreso y analizar sus intersecciones. Del análisis realizado se concluye que estas prácticas resultan sumamente variadas y revisten una alta significación numérica. Según se observa, más de 930 mil hogares (83\% de hogares en el primer quintil de ingreso) dispone de experiencia concreta o capacidades para la autogestión del hábitat en al menos una de las dimensiones identificadas. Asimismo, se observa que la movilización de redes de apoyo, la provisión comunitaria o autogestionada de servicios básicos, junto al mejoramiento y autoconstrucción de vivienda constituyen las modalidades más frecuentes. 



\section{*.*vis revistainvi}

\section{Introducción}

Entre la multiplicidad de reivindicaciones emergidas en Chile al alero de las movilizaciones sociales que alcanzaron su cenit en el así llamado "estallido social" de octubre de 2019, las demandas vinculadas a la realización efectiva del derecho a la vivienda y a un hábitat adecuados han tenido hasta ahora una figuración secundaria. Pese a que las organizaciones de pobladores han sido protagonistas visibles y relevantes de las manifestaciones y que los debates abiertos en torno al cambio constitucional han aludido frecuentemente a la omisión de referencias sobre dicho ámbito de derechos en el texto de la Constitución de 1980, la discusión sobre demandas relacionadas con la materialización de condiciones habitacionales dignas para el conjunto de la población se ha visto desplazada por otras reivindicaciones que han acaparado la atención pública (incluyendo, especialmente, aquellas asociadas a la previsión social, al acceso a ingresos y a la desigualdad en su distribución, además de demandas vinculadas a otras políticas sectoriales como salud y educación, además de la agenda de género).

No obstante, una serie de indicadores dan cuenta de la dimensión y profundidad que reviste aún la problemática habitacional en el país hacia fines de la década de 2010, existiendo escasas señales de progreso que destacar. La Agenda 2030 de Desarrollo Sostenible promovida por Naciones Unidas instaló entre sus metas la necesidad de asegurar el acceso de todas las personas a viviendas y servicios básicos adecuados, seguros y asequibles y mejorar los barrios marginales (ONU, 2015). Este desafío, de elevada complejidad, supone un conjunto variado de exigencias y reúne a numerosas demandas sociales cuyo denominador común se relaciona con la exclusión de importantes grupos de población derivada de condiciones deficitarias en materia de hábitat.

Teniendo presente este desafío, el diagnóstico oficial (Gobierno de Chile, 2019) estima en más de un millón cien mil hogares y en más de 3,5 millones de personas a la población residente en áreas urbanas que, hacia el año 2017, se encontraba privada de disfrutar de un hábitat adecuado. Ambas cifras respectivamente corresponden a un $21,9 \%$ de los hogares y a un $22,5 \%$ de la población urbana y evidenciaron un empeoramiento significativo respecto de la primera medición realizada en 2015, donde dichos valores eran de 18,4\% y 20,2\% (Gobierno de Chile, 2019).

Es relevante constatar que estas cifras sintetizan la confluencia de múltiples y agudas situaciones de carencia y vulneración de derechos, entre las que se incluyen la tenencia insegura de vivienda (7,0\% de hogares), hacinamiento $(8,4 \%)$, allegamiento $(4,1 \%)$, falta de servicios básicos adecuados $(2,9 \%)$, indisponibilidad de equipamientos y servicios sociales fundamentales como educación, salud y transporte en el entorno de la vivienda $(8,3 \%)$, junto a la presencia -en menor grado- de condiciones de extrema privación en materia de habitabilidad (vinculadas a la vivienda precaria o irrecuperable, tipología en la que habita un $0,5 \%$ de hogares) y de un elevado porcentaje de hogares $(9,2 \%)$ que, pese a ocupar viviendas de estándar adecuado, ve comprometida su seguridad económica por no disponer de acceso a una vivienda a un costo asequible (Ministerio de Desarrollo Social y Familia, 2018). Desde luego, cada una de estas formas de carencia o 


\section{*.*vis revistainvi}

vulneración de derechos (entre las que no se incluyen situaciones de extrema precariedad habitacional que afectan a zonas rurales ${ }^{1}$ ) se presentan en muchos casos de manera combinada, produciendo una experiencia compleja, estrechamente emparentada con la noción de pobreza multidimensional que actualmente funge como marco de referencia para la actuación de las políticas sociales (Beytía, 2016; Moreno, 2016).

En tanto, desde el punto de vista particular de la gestión de políticas habitacionales, los indicadores de déficit cuantitativo y cualitativo -que convencionalmente han sido empleados como medida de la cantidad de soluciones de vivienda a ser provistas por el Estado- tampoco exhiben mejoras. De acuerdo a las mediciones realizadas tanto con datos censales como con datos de la Encuesta Casen, el volumen de unidades de vivienda a proveer (déficit cuantitativo) alcanzaba hacia el año 2017 niveles que fluctuaban entre 400 y 500 mil, manteniendo una magnitud no lejana de la observada en mediciones realizadas durante la década anterior (Ministerio de Desarrollo Social y Familia, 2018).

En este cuadro, las necesidades vinculadas al allegamiento de hogares y de núcleos familiares -aunque revistan un carácter y composición diferentes a los de periodos anteriores- constituyen todavía el componente principal de la demanda insatisfecha por acceso a vivienda. Asimismo, el número de necesidades de mejoramiento de viviendas (déficit cualitativo) muestra un nivel sumamente elevado al año 2017, estimándose en una cifra cercana a 1,3 millones. Aunque dicha estimación comprende necesidades asociadas a diferentes grados de urgencia o complejidad, se observa que alrededor de un 30\% de estas necesidades corresponde a viviendas que presentan al menos dos de tres indicadores cualitativos deficitarios (sea por materialidad, espacio disponible y/o acceso a servicios básicos) (Ministerio de Desarrollo Social y Familia, 2018).

En paralelo, los altos costos de la vivienda se convierten en uno de los principales obstáculos que enfrentan las familias para acceder a una solución habitacional. Entre los años 2011 y 2017, el porcentaje de hogares propietarios en Chile descendió de 65,5\% a 60,4\%, de modo inverso al aumento evidenciado en la proporción de hogares arrendatarios (que subió de $17,0 \%$ a 21,9\%). En el mismo periodo, el porcentaje de hogares que pagaban un monto mensual de arriendo igual o superior a 0,2 UF por metro cuadrado se incrementó de un $11,7 \%$ a un $25,3 \%$, mientras que los hogares allegados aumentaron de 227 a 300 mil, identificándose que un $63,3 \%$ de los hogares que comparten vivienda lo hacen para generar ahorros o debido a que los ingresos con que cuenta el hogar no le permiten mantener una vivienda independiente. Asimismo, entre los años 2015 y 2017, el porcentaje de hogares que destinan más de un 30\% de su ingreso mensual al pago de arriendo o dividendo se elevó de un 8,8\% a un 10,6\% (Ministerio de Desarrollo Social y Familia, 2018).

Finalmente, se constata que un $17,3 \%$ de hogares no cuenta, simultáneamente, con equipamientos de educación y salud disponibles en el entorno próximo de la vivienda y tampoco con acceso cercano a un paradero o servicio de transporte, reduciendo ostensiblemente sus posibilidades de acceder a servicios indispensables y beneficiarse de las oportunidades disponibles en el territorio (Ministerio de Desarrollo Social y Familia, 2018).

$1 \quad$ El indicador oficial establecido para este fin, inserto en el marco del Objetivo $N^{\circ} 11$ de la Agenda 2030 y referido explícitamente a la situación de las ciudades, sólo considera a población urbana. 


\section{*.*vis revistainvi}

Teniendo en cuenta la elevada presión que estas múltiples formas de privación imponen a la política habitacional y el limitado repertorio de respuestas que ésta continúa ofreciendo (principalmente vinculadas a la asignación de subsidios para la adquisición de vivienda), se vuelve necesario reconsiderar el papel que desempeña en la actualidad la autogestión como instrumento alternativo para adecuar y/o promover mejoras a las condiciones deficitarias en materia de hábitat que afectan a significativos grupos de población que no logran acceder a los beneficios entregados por el Estado de manera oportuna. Tal como se observa, si las respuestas facilitadas desde las políticas y desde el mercado no han conseguido atenuar en una medida suficiente el volumen de estas demandas, es razonable suponer que numerosas familias y hogares no estén dispuestas a quedarse de brazos cruzados y aborden la gestión de soluciones habitacionales a través de sus propios recursos y poniendo en práctica distintas estrategias.

Si el camino principal del acceso a la vivienda y a un hábitat digno a través de la postulación a subsidios permanece bloqueado o no puede ser transitado de manera expedita por todos los grupos que demandan acceso a soluciones, ¿Qué es lo que pasa "al lado" de ese camino? ¿Qué formas de autogestión del hábitat existen en la actualidad y operan efectivamente como sustitutos o alternativas a la gestión de actores institucionales (públicos y privados) con el propósito de satisfacer necesidades de acceso a un hábitat adecuado?

Tal como se verá más adelante, estas estrategias son diversas y no se reducen a la autoconstrucción o la gestión particular de mejoras o transformaciones a las viviendas por sus ocupantes. Junto a estas formas de autogestión "clásicas" o "con las propias manos" -ampliamente reconocidas y discutidas en la literatura académica latinoamericana- es importante advertir la presencia de un conjunto de arreglos, prácticas y modalidades de autogestión del hábitat que contribuyen a fomentar el acceso a la vivienda adecuada o a reformar y mejorar la ya existente -optimizando sus atributos y la funcionalidad del stock habitacional - o, cuando menos, cumplen un papel significativo cuyo fin es mitigar las consecuencias y externalidades negativas que supone vivir en un hábitat deficitario.

En este sentido, el principal objetivo de este artículo apunta a actualizar la reflexión sobre el concepto de autogestión del hábitat, reclamando una ampliación de dicho concepto e intentando identificar sus expresiones más relevantes en el Chile actual. En atención a este plan, el segundo apartado presenta una discusión de los conceptos de autogestión del hábitat con referencia al marco del derecho a la vivienda adecuada. El tercer apartado propone una conceptualización contemporánea de la idea de autogestión del hábitat aplicada a la actual realidad chilena. El cuarto apartado, por su parte, presenta la metodología aplicada, mientras que el quinto desarrolla un análisis cuantitativo sobre la magnitud y distribución de las prácticas o estrategias de autogestión del hábitat con base en los datos de la Encuesta Casen 2017. Finalmente, en el sexto apartado, se presenta una breve conclusión y se levantan algunos desafíos para profundizar la investigación en la materia. 


\section{*.*vis revistainvi}

\section{Autogestión del hábitat y derecho a la vivienda adecuada}

El Marco Internacional del Derecho a una Vivienda Adecuada ("El derecho a una vivienda adecuada", 2010; Espejo, 2010) funge como principal referente al momento de establecer definiciones de orden normativo en torno a garantizar el acceso a condiciones dignas en materia de hábitat y vivienda. Señalando como hitos más relevantes las tres conferencias Hábitat realizadas a la fecha en Vancouver (ONU-Habitat, 1976), Estambul (ONU, 1996) y Quito (ONU-Habitat, 2017) y el Pacto Internacional de Derechos Económicos, Sociales y Culturales (ONU, 1966) -adoptado por Naciones Unidas en 1966 y que entró en vigor en 1976-, dicho marco reconoce a la vivienda en su calidad de satisfactor sinérgico, inserto en el territorio y cuya definición abarca no sólo a la vivienda como objeto o bien físico que cumple con determinados atributos materiales, sino que, además, comprende a la vivienda como bien físico que debe responder efectivamente a las necesidades y características socioculturales de sus ocupantes y proveer acceso a servicios básicos, equipamiento y condiciones salubres y seguras en su entorno (Espejo, 2010).

En términos de su operacionalización, el Marco Internacional del Derecho a una Vivienda Adecuada se expresa en siete criterios o características esenciales que las viviendas deben satisfacer a todo evento para considerarse adecuadas, a saber: (1) seguridad de la tenencia (la vivienda no es adecuada si sus ocupantes no cuentan con cierta medida de seguridad de la tenencia que les garantice protección jurídica contra el desalojo forzoso, el hostigamiento y otras amenazas); (2) disponibilidad de servicios (la vivienda no es adecuada si sus ocupantes no tienen agua potable, instalaciones sanitarias adecuadas, energía para la cocción, la calefacción y el alumbrado, y conservación de alimentos o eliminación de residuos); (3) asequibilidad (la vivienda no es adecuada si su costo pone en peligro o dificulta el disfrute de otros derechos humanos por sus ocupantes); (4) habitabilidad (la vivienda no es adecuada si no garantiza seguridad física o no proporciona espacio suficiente, así como protección contra el frío, la humedad, el calor, la lluvia, el viento u otros riesgos para la salud y peligros estructurales); (5) accesibilidad (la vivienda no es adecuada si no se toman en consideración las necesidades específicas de los grupos desfavorecidos y marginados); (6) ubicación (la vivienda no es adecuada si no ofrece acceso a oportunidades de empleo, servicios de salud, escuelas, guarderías y otros servicios e instalaciones sociales, o si está ubicada en zonas contaminadas o peligrosas); y (7) adecuación cultural (la vivienda no es adecuada si no toma en cuenta y respeta la expresión de la identidad cultural) ("El derecho a una vivienda adecuada", 2010).

En conformidad con esta definición -amplia y multidimensional-, tanto agencias internacionales como gobiernos, organizaciones de la sociedad civil y la academia, han adoptado el concepto de hábitat (o, más específicamente, hábitat residencial) como soporte articulador y categoría de gestión para procesar el conjunto de reivindicaciones relacionadas con el acceso a una vivienda adecuada y el disfrute de condiciones aptas en el entorno. El concepto de hábitat tiene como principal característica el reconocer la interacción dialéctica entre el territorio habitado y el sujeto habitante. Tal como se sugiere en Giraldo et al.: 


\title{
*.*vis revistainvi
}

\begin{abstract}
«(...) más allá de la ocupación física de un territorio, el hábitat constituye el referente simbólico y social en el que se localiza el ser humano de una manera multidimensional. Así, el hábitat significa algo más que tener un techo bajo el cual protegerse: es, en definitiva, tanto el espacio físico como la forma social y personal de apreciarlo y apropiarlo. En este sentido, lejos de ser algo homogéneo, simple y único, el hábitat cambia de un territorio -ciudad, región, nación- a otro, dependiendo de factores político-culturales, sociales, ambientales y económicos concretos» (Giraldo et al., 2006, p. 24-25).
\end{abstract}

Sin perjuicio de lo anterior, es importante consignar que el marco del derecho a la vivienda adecuada no establece fórmulas o soluciones únicas respecto a los actores a quienes les corresponde asumir responsabilidades en el proceso de provisión de vivienda y la producción del hábitat. Si bien este marco asigna un papel central al Estado en tanto depositario de obligaciones y encargado de velar por el ejercicio del derecho a la vivienda adecuada, se validan múltiples opciones en esta materia, que van desde un rol "facilitador" o promotor hasta un rol "garante" o proveedor a desempeñar por parte del Estado (sea como único responsable de la producción de vivienda, delegando enteramente dicha función en el sector privado y/o en la sociedad civil, o bien a través de fórmulas mixtas) ("El derecho a una vivienda adecuada", 2010).

En esta línea, es importante reconocer que tanto los individuos como colectivos organizados de la sociedad civil juegan un rol relevante junto al Estado y al sector privado en la producción del hábitat, lo que ha motivado el desarrollo de propuestas que enfatizan el carácter "social" de este proceso. Una de las formulaciones más conocidas al respecto ha sido postulada por Ortiz y Zárate (2002) y Ortiz (1998), quienes instalaron la idea de producción social del hábitat, apuntando específicamente al desarrollo de procesos autogestionados y no mercantiles de producción en los que cobra protagonismo la participación de la sociedad civil organizada. En su versión, la producción social del hábitat alude a una modalidad particular "(...) donde una organización de base de pobladores (cooperativas, asociaciones, mutual, sindicato, etc.), o una organización profesional no gubernamental (algunos de los tipos de ONG, centros de asistencia técnica, institutos populares de vivienda, asociaciones civiles pro-vivienda, etc.)" (Ortiz, 2002, citado en Rodríguez et al., 2007).

Tales organizaciones desarrollan la capacidad de producir viviendas y conjuntos habitacionales, orientados generalmente a sectores de bajos ingresos con capacidad de ahorro que participan activamente en todas las fases del proceso.

Esta perspectiva se emparenta a la nutrida tradición teórica y acción política generada en torno al concepto del "derecho a la ciudad", que reconoce la aspiración legítima de las y los habitantes urbanos por construir, decidir y crear condiciones adecuadas en las ciudades en las que habitan (Lefebvre, 1975). Como sostienen Rodríguez y Sugranyes (2017), el concepto del derecho a la ciudad ha dado pie a múltiples encarnaciones y representaciones; representaciones que oscilan entre tendencias utópicas, miradas ancladas a una matriz de derechos humanos y enfoques instrumentales o funcionales a procesos localizados de negociación entre actores e instituciones. La noción de autogestión del hábitat, en el sentido comentado más arriba, crece y se viabiliza a instancias de la exigencia de participación ciudadana como alternativa radical a la insuficiente capacidad de las políticas y del mercado. 


\section{*.*vis revistainvi}

No obstante, la Nueva Agenda Urbana -impulsada a instancias de la Conferencia Hábitat III realizada en Quito (2016)- declaró su respaldo a múltiples esquemas y modalidades de producción del hábitat, incluyendo soluciones cooperativas y modalidades mixtas de gestión en las que se incorpore la participación de la ciudadanía y, específicamente, el apoyo a "(...) planes de autoedificación y construcción gradual de viviendas, con especial atención a los programas de mejora de los barrios marginales y asentamientos informales" (ONU-Habitat, 2017). En dicho contexto, el mismo concepto ya aludido de producción social del hábitat es resignificado y despojado de su connotación de proceso autónomo o autogestionado, derivando en formas laxas de cooperación entre actores públicos, privados y de la sociedad civil, que estimula sinergias y externalidades positivas que se traducen no sólo en el fomento a la vivienda y el mejoramiento de condiciones en el entorno, sino también en la promoción del desarrollo económico (ONU-Habitat, 2017).

Al margen de distinciones analíticas y conceptuales, la discusión sobre la autogestión del hábitat y el rol de la comunidad en la producción de la vivienda ha sido un tópico recurrente y controvertido, siendo América Latina una de las regiones donde dicho debate adquirió mayor intensidad, especialmente desde mediados del siglo XX (Kozak, 2016; MacGuirk, 2015). Ante la constatación de la ineficacia del sector público para proveer de soluciones habitacionales a un ritmo sostenido y acorde al volumen de la demanda, agencias internacionales y prominentes arquitectos y planificadores impulsaron el desarrollo de esquemas alternativos en los que se buscaba incorporar de modo activo la participación popular en la producción del hábitat. Dichos esquemas -típicamente agrupados bajo la denominación de modelos de Auto-Ayuda / Auto-Construcción (AA / AC)- combinaban la necesidad de optimizar la asignación y administración de recursos económicos limitados con la promesa de canalizar la creatividad y resiliencia de las comunidades locales en la producción de soluciones pertinentes a los requerimientos y características socioculturales de los habitantes (Kozak, 2016).

Con el apoyo inicial de agencias crediticias y organismos financieros internacionales (como el Banco Mundial y el Fondo Monetario Internacional), los programas de AA/AC contribuyeron a transformar la imagen de precariedad y conflictividad -tradicionalmente vinculada a la participación popular en la gestión del hábitaten una herramienta potencialmente útil para encarar el cuantioso problema habitacional que afectaba a la región y fomentar la movilidad social ascendente (Kozak, 2016; MacGuirk, 2015). No obstante, los esquemas de AA / AC -que tuvieron su apogeo en las décadas de 1960 a 1980- declinaron en el contexto de la implantación de regímenes autoritarios y la introducción de reformas económicas neoliberales, siendo progresivamente sustituidos por esquemas que asignaron un rol primordial al mercado y donde la organización social de base fue desarticulada.

El caso de Chile, como destaca Gilbert (2002), tiene ribetes paradigmáticos al ser un precursor, en la región, de un modelo que asigna toda la responsabilidad de la producción de viviendas al sector privado, capitalizando las necesidades habitacionales de sectores de ingresos medios y bajos con el apoyo del Estado a través de subsidios a la demanda. Pese a esfuerzos e iniciativas que han intentado incorporar la participación como un componente relevante, los diseños programáticos y mecanismos de acceso a la vivienda con apoyo estatal han concedido un papel apenas secundario a la autogestión/autoproducción del hábitat. 


\section{*.*vis revistainvi}

Sin embargo, la idea de la autogestión del hábitat ha sido un pilar fundamental en las propias estrategias de subsistencia desplegadas por habitantes de asentamientos informales (Clichevsky, 2000) o precarios que, en el caso chileno, se han identificado convencionalmente con la figura de los campamentos;, así, persiste como formas estabilizadas de cooperación y resolución de problemas cotidianos que involucran no sólo el acceso y mejoramiento de la vivienda sino también el desarrollo de la calidad de vida barrial, el ejercicio de derechos y libertades y el acceso a oportunidades en el territorio.

Hoy cabe revisar el modo en que la persistencia de estas prácticas autogestionarias clásicas y la irrupción de otras nuevas o más difusas encarnan un cierto modelo de regeneración latinoamericano que, en oposición a los modelos instalados desde el Norte global, se caracterizan por soportar la participación de diferentes actores públicos y privados en acuerdos circunstanciales y flexibles (Paquette Vassalli, 2020), además de orientarse imperativamente al mejoramiento de las condiciones de vida de habitantes urbanos que enfrentan carencias y privaciones significativas por sobre objetivos de repoblamiento y de producción y captura de plusvalías. En esta línea, se considera pertinente revalorizar las prácticas de la autogestión del hábitat como mecanismo de resistencia a una cierta doctrina y/o normatividad de la regeneración urbana que hoy se instala como sentido común y único horizonte posible (Bustos-Peñafiel y Castrillo-Romón, 2020).

\section{Estrategias contemporáneas de autogestión del hábitat: coordenadas básicas para su conceptualización}

Las cifras sobre carencias habitacionales comentadas en la introducción de este artículo suelen ser interpretadas como expresión de una demanda insatisfecha que requiere de acciones urgentes por parte del Estado y del sector de la construcción, en estricto apego a los principios del Derecho a una Vivienda Adecuada. No obstante, pese a que todas estas carencias revelan necesidades agudas de mejora que pueden abordarse a través de diferentes programas o instrumentos, no se debe dejar de reconocer que, en muchos casos, estas representan simultáneamente modalidades o estrategias autónomas puestas en práctica por individuos y grupos de población con objeto de resolver (sea de manera provisoria o duradera) los problemas habitacionales que enfrentan, echando mano a los recursos físicos, materiales y financieros de los que disponen.

Desde esta mirada, la presencia de hogares allegados, por ejemplo -señalada actualmente como uno de los principales focos de demanda- puede ser resignificada también como una estrategia de uso compartido de la vivienda que tiene por objetivo asegurar el alojamiento de hogares que no disponen de una vivienda exclusiva, los cuales pueden tener relaciones de parentesco entre ellos y que puede implicar o no algún tipo de contraprestación o alguna forma de apoyo mutuo (Araos, 2008; Moreno, 2012). Por otra parte, la así denominada vivienda precaria o "irrecuperable", no sólo es una solución sub-estándar en términos de sus condiciones de materialidad o habitabilidad, sino también es una respuesta contingente al problema del acceso a la vivienda que, aunque no satisfaga los requisitos mínimos de una vivienda adecuada -de acuerdo a la 


\section{*.*vis revistainvi}

definición normativa- es una modalidad de vivienda autoconstruida que emplea técnicas artesanales y utiliza los materiales que los propios hogares han sido capaces de recolectar, disponer y combinar (principalmente, material de desecho o reutilizado).

A fin de precisar variantes y evitar confusiones terminológicas, Rodríguez et al. (2007) propusieron una tipología refinada que busca separar modalidades y significados particulares vinculados al concepto de producción social del hábitat. Entonces, en un primer escalón se identificaría el concepto de autoproducción, que engloba de modo general a "(...) todos los procesos de producción que se realizan bajo iniciativa y control de los propios usuarios, de manera: i) individual/familiar, ii) comunitaria, colectiva y organizada" (Rodríguez et al., 2007, p. 17).

Dentro de esta categoría, se admiten variadas formas de movilización de recursos privados y/o comunitarios, entendiéndose que la autoproducción del hábitat emerge como un patrón transversal a diversos grupos socioeconómicos (incluyendo también a estratos medios y altos) y se traduce en múltiples formas de solución (incluyendo la autoconstrucción, la "vivienda por encargo" y también modalidades cooperativas de reforma o producción de hábitat que abarcan tanto el espacio público como el privado).

Luego, en un sentido más restringido, los autores citados abordan en términos singulares el concepto de autogestión del hábitat el que, según sostienen: “(..) se vincula de manera más específica con formas de producción del hábitat colectivas y organizadas, sostenidas por organizaciones sociales que persiguen en forma explícita el desarrollo de distintos tipos de procesos políticos de construcción de poder popular" (Rodríguez et al., 2007, p. 18).

Pese a esta relevante precisión, presuponer una organización o la construcción potencial de un proyecto político como requisito indispensable para identificar un proceso de autogestión del hábitat implica un umbral tal vez demasiado exigente. Sobre todo, teniendo en cuenta la espontaneidad e inestabilidad a la que pueden estar sujetas ciertas prácticas que, siendo capaces de movilizar recursos y producir organización de base, no necesariamente plantean una proyección política de su acción. En este sentido, puede ser relevante considerar un significado más acotado de autogestión del hábitat, vinculado especialmente a la capacidad de producir organización de base y gestionar recursos de modo colectivo.

Alineada con esta perspectiva, a partir de la discusión de la definición del componente organizativo de los campamentos en Chile, Rivas plantea la noción de autogestión comunitaria entendida como el "(...) proceso mediante el cual se desarrolla la capacidad de una comunidad para identificar los intereses o necesidades que le son propios y que no se encuentran satisfechas por mecanismos exógenos a la comunidad" (Rivas, 2013, p. 30).

La autora destaca, además, que dicha capacidad es, a la vez, organizadora y busca satisfacer esas necesidades en la práctica cotidiana a través de acciones colectivas.

Adicionalmente a la tensión entre un significado amplio o más restringido de autogestión del hábitat, otro problema se refiere a la identificación unívoca entre autogestión y autoconstrucción. En este plano, la autoconstrucción señala la forma más sobresaliente o reconocida de autogestión del hábitat, siendo definida como "(...) la práctica de edificar la vivienda o los componentes del hábitat por sus propios usuarios" (Rodríguez 


\section{*.*vis revistainvi}

et al., 2007, p. 19). Finalmente, también se reconoce a la producción dirigida o facilitada por organismos civiles especializados sin fines de lucro como otra modalidad singular de producción social del hábitat.

Conforme a las definiciones ya discutidas, dichas prácticas implican alguna forma de autonomía en la gestión del hábitat. Aun cuando esta autonomía pueda no ser un resultado deseado o buscado por los propios hogares (surgiendo como respuesta forzosa a la desatención pública de sus demandas o a la insuficiencia o inequidad en la distribución de los recursos) y las soluciones que genere no permitan cumplir con exigencias normativas básicas en términos del acceso a una vivienda adecuada, son relevantes en términos de observar vías alternativas y auto-sustentadas al "camino" principal ofrecido por las soluciones que el Estado y/o el mercado puedan facilitar.

En muchos casos, como se ha visto, tales prácticas pueden no encajar con una noción o idea "clásica" de autogestión del hábitat en el sentido de articularse con procesos colectivos que emplean la autonomía como forma de lucha y que implican organización social de base y alguna forma de proyección política o reivindicativa. Teniendo en cuenta lo anterior, es pertinente señalar que la autonomía es una característica necesaria pero no suficiente para identificar procesos autogestionados de hábitat.

Buscando un punto medio entre una definición demasiado amplia (donde caben todas las formas de autoproducción o autoprovisión del hábitat) y una definición demasiado estricta (donde la organización política y la resistencia son las únicas formas de autogestión del hábitat que interpretarían correctamente tal concepto), se opta por considerar la dimensión ya aludida de resolución comunitaria de problemáticas habitacionales, que implica un diagnóstico y una acción colectiva orientada a resolver necesidades de un conjunto de personas u hogares que comparten una situación y que tiene como principal fuente de recursos el capital (físico, social y financiero) acumulado por la comunidad, operando en independencia o "al lado" del camino institucional.

\section{Metodología}

Siguiendo estas orientaciones conceptuales, a continuación se aborda el desafío de catalogar y describir algunas de las principales expresiones que reviste en la actualidad la autogestión del hábitat en Chile. A ello se suma el propósito de establecer una cuantificación somera del número de hogares que desarrolla formas o prácticas de autogestión del hábitat.

Ambos desafíos no se encuentran exentos de dificultades, pues los procesos de autogestión del hábitat son dinámicos y se oponen, por definición, a cualquier intento de categorización, normalización o estandarización estadísticas. Sin embargo, se afirma la posibilidad de efectuar un acercamiento panorámico y descriptivo que permita, aun con cierto incertidumbre o imprecisión, aproximarse a estimar la magnitud de sus principales manifestaciones. 


\section{*.*vis revistainvi}

Para este propósito, se consideró una metodología cuantitativa y descriptiva basada en el procesamiento y desarrollo de inferencia estadística conforme al uso de los datos recolectados por la Encuesta de Caracterización Socioeconómica Nacional (Casen) 2017. Se da prioridad a esta fuente de datos por corresponder a un instrumento estadístico de cobertura y representatividad nacional. En contraste con otras fuentes disponibles - como el Censo de Población y Vivienda- el cuestionario de la Encuesta Casen incluye un amplio repertorio de variables que facilitan la caracterización de las modalidades particulares a través de las que los hogares acceden a vivienda (incluyendo información detallada sobre formas de tenencia y financiamiento), además de recoger una serie de atributos relativos a los servicios disponibles, al entorno y la proximidad a equipamientos, junto con identificar acciones realizadas o planificadas por los hogares con el objetivo de mejorar sus condiciones habitacionales. Asimismo, dispone de variables de ingreso y caracterización socioeconómica que resultan de utilidad para encuadrar al conjunto de hogares que practican diferentes estrategias de autogestión del hábitat.

Las estimaciones elaboradas (que se detallan en la siguiente sección), apuntan al objetivo de establecer una magnitud mínima (o "piso") de hogares que han desarrollado alguna capacidad o cuentan con experiencia relevante de autogestión del hábitat. De esta forma, sólo para efectos de no sobreestimar el tamaño de los grupos de población involucrados y de no incurrir en la identificación de "falsos positivos" (hogares que declaran realizar alguna modalidad de autogestión, sea por opción voluntaria o por la autosuficiencia que le concede el capital económico acumulado), se adopta la decisión de considerar como foco de atención prioritario a la población perteneciente a hogares del primer quintil de ingreso autónomo per capita (20\% de menor ingreso).

Considerando que el ingreso per cápita mensual en dicho segmento era, al año 2017, inferior a $\$ 99.985^{2}$.- y que la capacidad de ahorro y consumo, por tanto, es sumamente reducida, es razonable suponer que dicho grupo debe recurrir a estrategias de autogestión o provisión por medios alternativos al mercado para satisfacer necesidades y realizar inversiones que les permitan materializar mejoras en sus condiciones de vida.

No obstante $-y$ pese a las justificaciones señaladas-, se recalca que esta definición resulta completamente arbitraria, pues algunas de las prácticas que se describen a continuación se extienden también a otros grupos socioeconómicos, situación que es reconocida también por el Estado que también prioriza dentro de sus políticas y programas a población de sectores de ingreso medio-bajo y medio que despliegan estrategias informales o autogestionadas de gestión de hábitat.

De manera excepcional, para dimensionar algunas expresiones de autogestión que no son adecuadamente recogidas por los datos de la encuesta Casen (sea por razones de diseño muestral o ausencia de información relevante), el diagnóstico es complementado con datos procedentes de otras estadísticas públicas.

2 Véase datos publicados por Ministerio de Desarrollo Social y Familia (2017). 


\section{Diagnóstico cuantitativo de las prácticas de autogestión del hábitat en Chile en el umbral de la década de 2020}

Conforme a la metodología propuesta, en la presente sección se expone un listado sumario de las principales expresiones que reviste la autogestión del hábitat con datos de la Encuesta Casen 2017.

El listado presentado no pretende ser exhaustivo, pero si busca fijar algunas categorías y magnitudes sobre las cuales es posible profundizar e indagar posteriormente con mayor detalle a través de otras estrategias metodológicas.

\section{a. Autoconstrucción de vivienda}

La autoconstrucción es una práctica profundamente enraizada y persistente en Chile. Aunque no existen estadísticas que permitan cuantificar su incidencia con exactitud, es relevante subrayar que, según datos de la Encuesta Casen 2017, entre los hogares pertenecientes al primer quintil que son ocupantes de una vivienda en propiedad, un 31\% (equivalente a más de 230 mil hogares) manifiesta haber adquirido la vivienda sólo con sus propios recursos, sin haber tenido subsidio estatal, sin haber contraído algún crédito o préstamo y sin mantener alguna deuda asociada (excluyendo, además, a departamentos en edificio y viviendas recibidas como herencia o traspaso gratuito).

Esta estrategia tiene, asimismo, un especial arraigo entre hogares residentes en zonas rurales (más del $40 \%$ de los hogares del primer quintil que adquirieron o produjeron la vivienda en que habitan "sólo con sus propios recursos", residen en zona rural).

La autoconstrucción, asimismo, ha adquirido notoriedad como modalidad de respuesta expedita a desastres naturales que han derivado en la destrucción o daño irreparable de vivienda (anticipándose a o sustituyendo soluciones habitacionales "definitivas" provistas con apoyo de subsidios estatales). Otro punto a considerar es que la autoconstrucción puede concretarse en sitios compartidos por dos o más viviendas (más del 25\% de los hogares de bajos ingresos que dispone de una vivienda propia adquirida solo con sus recursos corresponde a ocupantes de casas en sitios con más de una construcción en su interior, por lo que también la autoconstrucción se asociaría a una forma de densificación predial autogestionada) y que están o no regularizados en términos de su situación de inscripción o tenencia.

\section{b. Cesión y uso compartido de vivienda}

Un fenómeno de elevada incidencia en el país corresponde a la cesión de vivienda y/o al allegamiento y/o uso compartido de vivienda, sin comprometer ningún tipo de pago y, en principio, con la aprobación del propietario/a o dueño/a. 


\section{*.*vis revistainvi}

Si se considera nuevamente al primer quintil de ingresos, el tamaño del primer grupo podría estimarse en una cifra superior a los 175 mil hogares, correspondiente a más del 15\% de hogares de dicho quintil. En el caso de los hogares que comparten vivienda con otros hogares y no realizan ningún tipo de pago por residir en dicha condición, se podría estimar su presencia (una parte de los cuales corresponde a hogares identificados dentro de la categoría del allegamiento externo) en una magnitud superior a los 50 mil hogares ${ }^{3}$.

Cabe precaver que estos grupos también tienen una presencia numerosa entre otros segmentos socioeconómicos, por lo que dichas cifras podrían estar subestimadas. No obstante, también se abre la interrogante de indagar en la presencia de algún tipo de organización colectiva asociada a esta forma de gestión o provisión de vivienda, la que podría tener un patrón mucho más disperso, tanto en términos territoriales como en términos de los mecanismos que la sostienen.

\section{c. Autogestión comunitaria de suelo (campamentos) y/o de vivienda vacante o sin uso}

Aunque evidentemente subreportadas o subdeclaradas, existen modalidades de ocupación de suelo y vivienda que se realizan informalmente; éstas, que terminan quedando sin regularizar o fuera de la legalidad desde el punto de vista de la tenencia, se dan de modo esporádico o continuo en el tiempo.

Desde luego, en el caso de Chile, una de las principales expresiones de este fenómeno remite a los campamentos, cuya definición oficial se establece en consistencia con criterios internacionales para la identificación de asentamientos precarios o informales y que refiere, específicamente a

"Asentamientos precarios de ocho o más hogares que habitan en posesión irregular un terreno, con carencia de al menos uno de los tres servicios básicos (electricidad, agua potable y sistema de alcantarillado), y cuyas viviendas conforman una unidad socio territorial definida» (Ministerio de Vivienda y Urbanismo, 2019, p. 7).

En relación a los campamentos, la última actualización del catastro realizado por el Ministerio de Vivienda y Urbanismo reportó un total de 802 campamentos, asentamientos en los cuales viven 47.050 hogares y una población estimada superior a 140 mil habitantes ${ }^{4}$.

Esta medición es, de momento, la aproximación más exhaustiva y actualizada existente acerca de este fenómeno. Por otra parte, los datos de la Encuesta Casen 2017 ya identificaban que alrededor de un 1,3\% de hogares del primer quintil de ingresos (14 mil hogares, aproximadamente) declaraba habitar viviendas en condición de ocupación o posesión irregular. Si se considera a las viviendas emplazadas en sitios ocupados en esta misma condición (independientemente de la situación de vivienda), dicha estimación podría elevarse a un 1,7\% (19 mil hogares). En ambos casos, es importante aclarar que esta estimación se basa exclusivamente

3 Esta estimación considera exclusivamente a hogares que comparten viviendas y cuya situación de tenencia corresponde a cesión por familiar u otra persona. El número total de hogares allegados en Chile se estimaba al año 2017 en una cifra superior a los 300 mil hogares. Tampoco se incluye a núcleos familiares secundarios (allegamiento interno, definido por la presencia de unidades familiares secundarias que no conforman hogares en sentido estadístico), cuya magnitud ascendía, en el mismo año, a 155 mil hogares.

4 Información publicada por el Ministerio de Vivienda y Urbanismo, (s. f.) 


\section{*.*vis revistainvi}

en el reporte de los propios hogares, para los cuales podrían existir aprensiones respecto de reconocer una situación de tenencia irregular.

La ocupación colectiva de suelo de modo irregular, ampliamente investigada para el caso de los campamentos, supone quizás la forma más radical de autogestión del hábitat e íntimamente asociada a las representaciones de la pobreza extrema empleadas instrumentalmente por la política pública (Abufhele, 2019) Dicha modalidad de autogestión del hábitat a menudo implica también una urbanización autónoma y espontánea (que puede consolidarse a través del tiempo) y la producción comunitaria de servicios, equipamientos y de espacios públicos, en la medida en que no existen opciones de radicación y/o de regularización de la tenencia. Estos procesos, además, desafían una serie de obstáculos y amenazas relacionadas con las condiciones físicas y medioambientales del suelo ocupado, que en muchos casos corresponden a zonas de riesgo y/o expuestos a focos de contaminación (Rivas, 2013).

La ocupación irregular también puede incluir inmuebles deshabitados o que no tienen un uso habitacional, debido a diferentes motivos. Tal como fue revelado por el Censo 2017, más de 600 mil viviendas en el país no tenían moradores o se encontraban en arriendo, en venta, en situación de abandono u otra condición (descontando viviendas con uso de temporada, vacacional o de otro tipo). Por su magnitud, la gestión del stock vacante de vivienda se convierte también en una problemática relevante en el país y supone también eventuales conflictos a futuro por su uso y apropiación, sobre todo cuando se trata de viviendas que no están incorporadas en el mercado de venta y arriendo.

\section{d. Autogestión de mejoras y/o ampliaciones a la vivienda}

Un componente de autogestión al que comúnmente se presta escasa atención es el referido a la mejora y/o transformación de la vivienda. Según la Encuesta Casen 2017, dentro del primer quintil de ingresos, uno de cada cinco hogares (21\%, equivalente a más de 230 mil hogares) había realizado alguna mejora o transformación a su vivienda en los últimos dos años empleando para ello sólo recursos propios (sin subsidio estatal).

Se destaca asimismo que, dentro de este grupo, el grueso de las mejoras o transformaciones efectuadas son de gran envergadura y complejidad, resaltando la realización de reparaciones estructurales de muros, techo y/o piso (53\%) y ampliaciones o construcción de piezas (23\%), a las que se suma la construcción de tabiques interiores, revestimientos, reparaciones no estructurales y otras terminaciones (16\%).

Aunque en este tipo de acciones la gestión particular pueda ser una estrategia predominante, no debe descartarse el apoyo de redes de contactos que puedan facilitar el acceso a materiales de construcción o prestar mano de obra sin mediar pagos en dinero. Tal como la propia encuesta Casen rescata, un $41 \%$ de hogares del primer quintil de ingresos manifiesta poder contar con una persona ajena al hogar que podría ayudarle a realizar reparaciones en él (gasfitería, mecánica, albañilería, reparación de artículos eléctricos), otro 23\% señala contar con una persona en el propio hogar que podría hacerlo y un $11 \%$ declara disponer tanto de un apoyo externo como interno al hogar que podría ejercer dichas tareas. 


\section{*.*vis revistainvi}

Las ampliaciones y/o construcción de recintos, específicamente, son obras que también pueden favorecer la provisión de alojamiento para otras personas u hogares o bien pueden contribuir a generar ingresos para el hogar en la medida que permiten el desarrollo de actividades productivas, comerciales y/o el arrendamiento de piezas.

\section{e. Provisión comunitaria o irregular de servicios básicos}

El acceso formal a los servicios básicos (agua, gas y electricidad, principalmente) representa un alto costo para los hogares de menor ingreso, forzando a gestionar modalidades alternativas para su provisión o bien accediendo de modo irregular a ellos.

Teniendo en cuenta las dificultades asociadas a establecer la magnitud de este fenómeno, al menos puede constatarse que existe una significativa frecuencia de hogares que accede a los servicios de agua y electricidad de maneras no reguladas o compartiendo el abastecimiento y/o el pago con otros hogares. Un dato de interés al respecto es que alrededor de 10 mil hogares en el primer quintil (alrededor de un 1\% del total de dicho grupo socioeconómico) declara acceder a agua o energía eléctrica sin contar con medidor en su vivienda. Complementariamente, más de 10\% de hogares en el mismo quintil (del orden de 110 a 120 mil hogares) consumen agua o energía eléctrica de la red pública empleando un medidor compartido con otros hogares.

Estas formas de acceso a servicios básicos, aunque puedan ser regulares, denotan la existencia de algún tipo de organización que favorece el aprovechamiento colectivo o bien la cofinanciación del acceso.

Junto a estas modalidades, también se pueden destacar las formas de organización autogestionada para la provisión de servicios básicos que surgen en el contexto de los campamentos y de localidades rurales aisladas que carecen de cobertura por parte de las empresas proveedoras y/o de soluciones alternativas que puedan ser provistas por el Estado y/o los gobiernos locales.

En el caso del agua, modalidades de organización comunitaria vinculadas al acarreo o el suministro a través de camiones aljibe han tendido a ampliarse en atención a la escasez hídrica que afecta a importantes cuencas y zonas del Norte, Centro y Sur del país.

Sumando todas las modalidades descritas, es posible arribar a una estimación superior a los 260 mil hogares que despliegan estrategias autogestionadas o semi-autogestionadas de acceso a servicios básicos,

\section{f. Redes de apoyo para el mejoramiento del hábitat}

Las redes de apoyo, entendidas como redes de contactos informales entre personas que se activan a fin de satisfacer necesidades y proveer de ayuda en casos de emergencia, son un factor de especial relevancia para sostener y fomentar la mejora en las condiciones de un hábitat autogestionado. 


\section{*.*vis revistainvi}

Tal como ya se comentó en el caso de las mejoras a la vivienda, la existencia de contactos puede contribuir a reducir costos y facilitar la provisión de servicios que los hogares no pueden adquirir en el mercado o a los que no pueden asignar prioridad debido al escaso presupuesto con el que cuentan.

Asimismo, otro problema dice relación con la distancia física, la falta de transporte o de competencias específicas que pueden constituirse en una importante barrera para disponer de recursos relevantes para los hogares.

En esta línea son importantes las economías locales del cuidado, destacando la posibilidad de contar con apoyos para atender necesidades de personas con discapacidad, enfermas o con algún grado de dependencia física y, de tal modo, permitir que otras personas puedan insertarse en el mercado laboral, estudiar o participar de actividades sociales, recreativas y comunitarias.

Según es mostrado con información de Casen 2017, un 57\% de hogares en el primer quintil (casi 640 mil hogares) conoce personas externas al hogar que podrían ayudarles para resolver cuatro o más situaciones cotidianas en las que sus integrantes podrían requerir de ayudas, incluyendo el cuidado de personas enfermas, niños/as o personas con alguna discapacidad, el acceso a un vehículo, dinero u apoyo para conseguir un empleo en caso de requerirlo, la asistencia para realizar trámites legales o financieros, el uso de tecnologías o la realización de reparaciones en el hogar (incluyendo gasfitería, mecánica, albañilería o reparación de artículos eléctricos (todos recursos sociales altamente valorados y que contribuyen de manera directa o indirecta a la autogestión del hábitat).

En último término, también se manifiesta la importancia de redes de apoyo que favorezcan la vinculación de individuos o colectivos vulnerados y/o que enfrentan condiciones agudas de deterioro, como es el caso de las personas en situación de calle, cuya presencia se estima en 15.208 personas según los datos del Registro de Personas en Situación de Calle del Ministerio de Desarrollo Social y Familia actualizados al mes de noviembre de 2019.

\section{g. Síntesis}

En la Tabla 1, finalmente, se expone el recuento de todas las prácticas reseñadas y se elabora una estimación sintética que identifica al conjunto de hogares del primer quintil de ingreso que desarrollan autogestión o tienen capacidad de movilizar una o más de estas distintas estrategias. Según se constata, el uso de capital social es aquella capacidad de autogestión más extendida entre dichos hogares $(56,8 \%$, alcanzando a casi 640 mil hogares). Sin perjuicio de lo anterior, la autoconstrucción o autoprovisión de vivienda, la autogestión de mejoras y ampliaciones y la provisión comunitaria o autogestionada de servicios básicos alcanzan una magnitud sumamente relevante (cada una de estas modalidades representa entre los 220 y 260 mil hogares).

Sumando el uso de cada una de estas estrategias (ver Tabla 1), se tiene que un $83 \%$ de hogares (más de 930 mil hogares) del primer quintil dispone de experiencia concreta o capacidades para la autogestión del hábitat, mientras que una cifra cercana a 147 mil hogares practica la autogestión en a lo menos tres de las seis dimensiones abordadas en este documento. 


\section{* revistainvi}

Tabla 1.

Estimación absoluta y relativa de la incidencia de prácticas de autogestión del hábitat en hogares chilenos (2017).

\begin{tabular}{|c|c|c|c|c|}
\hline Dimensión & Indicador & Número & $\begin{array}{c}\text { Porcentaje respecto } \\
\text { de hogares del primer } \\
\text { quintil }\end{array}$ & $\begin{array}{l}\text { Porcentaje } \\
\text { respecto del total } \\
\text { de hogares }\end{array}$ \\
\hline $\begin{array}{l}\text { 1. Autoconstrucción o } \\
\text { autoprovisión de vivienda }\end{array}$ & $\begin{array}{l}\text { 1. Hogares que adquirieron vivienda } \\
\text { sólo con sus propios recursos }\end{array}$ & 232.420 & 20,6 & 4,0 \\
\hline \multirow{3}{*}{$\begin{array}{l}\text { 2. Cesión y uso compartido de } \\
\text { vivienda }\end{array}$} & $\begin{array}{l}\text { 2. Hogares que ocupan vivienda } \\
\text { cedida o comparten vivienda sin pago } \\
\text { en dinero }\end{array}$ & 226.630 & 20,1 & 3,9 \\
\hline & $\begin{array}{l}\text { 2.1. Hogares que son ocupantes } \\
\text { principales de la vivienda en situación } \\
\text { de cesión por familiar u otro }\end{array}$ & 176.061 & 15,6 & 3,0 \\
\hline & $\begin{array}{l}\text { 2.2. Hogares que comparten vivienda } \\
\text { con otros hogares y no realizan } \\
\text { ningún tipo de pago por servicios de } \\
\text { vivienda }\end{array}$ & 50.569 & 4,5 & 0,9 \\
\hline \multirow{3}{*}{$\begin{array}{l}\text { 3. Autogestión comunitaria de } \\
\text { suelo y ocupación de vivienda } \\
\text { vacante o sin uso }\end{array}$} & $\begin{array}{l}\text { 3. Hogares que habitan viviendas o } \\
\text { sitios ocupados o poseídos de manera } \\
\text { irregular }\end{array}$ & 19.004 & 1,7 & 0,3 \\
\hline & $\begin{array}{l}\text { 3.1 Hogares que habitan viviendas en } \\
\text { condición de ocupación o posesión } \\
\text { irregular }\end{array}$ & 14.841 & 1,3 & 0,3 \\
\hline & $\begin{array}{l}\text { 3.2. Hogares que habitan viviendas } \\
\text { localizadas en sitios con ocupación o } \\
\text { posesión irregular }\end{array}$ & 19.004 & 1,7 & 0,3 \\
\hline $\begin{array}{l}\text { 4. Autogestión de mejoras y/o } \\
\text { ampliaciones a la vivienda }\end{array}$ & $\begin{array}{l}\text { 4. Hogares que realizaron alguna } \\
\text { mejora o transformación a su } \\
\text { vivienda en los últimos dos años sólo } \\
\text { con recursos propios (sin subsidio } \\
\text { estatal). }\end{array}$ & 231.071 & 20,5 & 4,0 \\
\hline
\end{tabular}




\section{* revistainvi}

\begin{tabular}{|c|c|c|c|c|}
\hline Dimensión & Indicador & Número & $\begin{array}{c}\text { Porcentaje respecto } \\
\text { de hogares del primer } \\
\text { quintil }\end{array}$ & $\begin{array}{l}\text { Porcentaje } \\
\text { respecto del total } \\
\text { de hogares }\end{array}$ \\
\hline \multirow{4}{*}{$\begin{array}{l}\text { 5. Provisión comunitaria o } \\
\text { autogestionada de servicios } \\
\text { básicos }\end{array}$} & $\begin{array}{l}\text { 5. Hogares que acceden a agua o } \\
\text { electricidad de manera autogestionada } \\
\text { o compartida }\end{array}$ & 260.296 & 23,1 & 4,5 \\
\hline & $\begin{array}{l}\text { 5.1. Hogares que acceden a agua } \\
\text { o energía eléctrica sin contar con } \\
\text { medidor en su vivienda }\end{array}$ & 18.887 & 1,7 & 0,3 \\
\hline & $\begin{array}{l}\text { 5.2. Hogares que acceden a agua o } \\
\text { energía eléctrica mediante medidor } \\
\text { compartido con otros hogares }\end{array}$ & 149.985 & 13,3 & 2,6 \\
\hline & $\begin{array}{l}\text { 5.3. Hogares que acceden a agua o } \\
\text { energía eléctrica mediante fuente } \\
\text { alternativa a red pública }\end{array}$ & 91.424 & 8,1 & 1,6 \\
\hline $\begin{array}{l}\text { 6. Activación de redes de apoyo } \\
\text { para el mejoramiento del hábitat }\end{array}$ & $\begin{array}{l}\text { 6. Hogares que cuentan con redes } \\
\text { de apoyo para acceder a servicios } \\
\text { o satisfacer necesidades de manera } \\
\text { alternativa }\end{array}$ & 639.636 & 56,8 & 11,0 \\
\hline \multicolumn{2}{|c|}{$\begin{array}{l}\text { Desarrolla autogestión o cuenta con capacidad de autogestión en al menos } \\
\text { una dimensión }\end{array}$} & 934.011 & 82,9 & 16,1 \\
\hline \multicolumn{2}{|c|}{ Cuenta con capacidad de autogestión sólo en una dimensión } & 437.279 & 38,8 & 7,5 \\
\hline \multicolumn{2}{|c|}{ Cuenta con capacidad de autogestión en dos dimensiones } & 350.193 & 31,1 & 6,0 \\
\hline \multicolumn{2}{|c|}{ Cuenta con capacidad de autogestión en tres o más dimensiones } & 146.539 & 13,0 & 2,5 \\
\hline
\end{tabular}

Fuente: elaboración propia en base a datos de la Encuesta Casen 2017, Ministerio de Desarrollo Social y Familia. 


\section{*.*vis revistainvi}

\section{Conclusión}

Iniciando la década de 2020, las estrategias y prácticas de autogestión del hábitat en Chile están lejos de desempeñar un papel marginal. En esta investigación se ha verificado, incluso desde las estimaciones más conservadoras, su presencia en centenares de miles de hogares, siendo ésta una cantidad que podría amplificarse con facilidad en la medida que se extiende su definición operacional, abarcando manifestaciones directa e indirectamente relacionadas e involucrando una mayor variedad de grupos socioeconómicos.

Pero, junto con reconocer su relevancia numérica y su transversalidad, se constata la necesidad de evitar una asociación unidireccional entre hábitat autogestionado y algunas expresiones singulares que, por su tradición y notoriedad, tienden a acaparar la atención en el debate político-sectorial; tal es el caso de los campamentos (que aquí se ha insertado en un concepto más amplio de autogestión comunitaria del suelo) y el de la autoconstrucción de vivienda (que, según lo discutido, responde a una entre otras muchas herramientas de autogestión del hábitat).

El hábitat autogestionado muestra una cara diversa y versátil: allí donde el Estado y el mercado no consiguen producir las soluciones requeridas en cuanto a oportunidad, cantidad y velocidad, son los hogares (pese a unos ingresos y una capacidad de movilizar recursos extremadamente limitados) quienes enfrentan la urgencia con decisión, asociándose entre sí e implementando respuestas que, aunque provisorias e insuficientes en calidad, permiten parcialmente mitigar las necesidades de sus integrantes y fomentar mejoras en su bienestar.

Por otra parte, aunque débilmente visibilizadas, se constata que la mayor parte las modalidades de gestión autónoma del hábitat que se han descrito en este documento tienen una dilatada historia y forman parte del repertorio de recursos que los hogares activan cotidianamente y están estrechamente conectados con las densas redes sociales de que disponen los hogares de bajos ingresos y que entregan protección en momentos de crisis (Moser, 1998). En el contexto del actual proceso político que vive el país y teniendo como referencia el Marco del Derecho a la Vivienda Adecuada, comprender estos mecanismos resulta fundamental a fin de dibujar los bordes y evaluar las capacidades efectivas con los que cuenta el Estado y el mercado para hacer frente a la problemática de la vivienda y del hábitat en general.

En última instancia, se advierte la necesidad por profundizar en la investigación de algunas modalidades específicas de autogestión del hábitat y producir conocimiento sistemático en torno a ellas. En particular, se constata la falta de estudios sobre las iniciativas de mejoras y ampliaciones a viviendas, tema que sólo colateralmente ha sido explorado en la investigación académica y que tiene suma relevancia, dada la elevada cifra que exhibe actualmente el déficit cualitativo de vivienda. Otros temas que ameritan una mayor discusión remiten a la relevancia que poseen las redes de apoyo y su relación con el desarrollo de procesos de autoconstrucción y mejora del hábitat, particularmente en el caso de zonas rurales y pequeñas localidades. 


\section{Referencias bibliográficas}

Abufhele, V. (2019). La política de la pobreza y el gobierno de los asentamientos informales en Chile. EURE, 45(135), 49-69. https://doi.org/10.4067/ S0250-71612019000200049

Araos, C. (2008). La tensión entre filiación y conyugalidad en la génesis empírica del allegamiento: estudio cualitativo comparado entre familias pobres de Santiago de Chile. (Tesis Magíster en Sociología, sin publicar). Pontificia Universidad Católica de Chile, Santiago, Chile.

Beytía, P. (2016). La estructura interna de la pobreza multidimensional. En C. Siles (Ed.), Los invisibles. Por qué la pobreza y la exclusión social dejaron de ser prioridad (pp. 71-88). Instituto de Estudios de la Sociedad.

Bustos-Peñafiel, M. y Castrillo-Romón, M. (2020). Luces y sombras de la regeneración urbana: perspectivas cruzadas desde Latinoamérica y Europa. Revista INVI, 35(100), 1-19. https://doi.org/10.4067/ S0718-83582020000300001

Clichevsky, N. (2000). Informalidad y segregación urbana en América Latina: una aproximación. CEPAL.

El derecho a una vivienda adecuada. (2010). Folletos informativos sobre los derechos humanos, (21). http://www.ohchr.org/Documents/Publications/ FS21 rev 1 Housing sp.pdf

Espejo, N. (2010). El derecho a una vivienda adecuada. Revista CIS, 8(13), 48-63.

Gilbert, A. (2002). Power, ideology and the Washington consensus: the development and spread of Chilean housing policy. Housing Studies, 17(2), 305-324. https://doi.org/10.1080/02673030220123243

Giraldo, F., García, J., Bateman, A., y Alonso, A. (2006). Hábitat y pobreza: los objetivos de desarrollo del milenio desde la ciudad. ONU-Hábitat.
Gobierno de Chile. (2019). Segundo informe nacional voluntario: Agenda 2030 para el Desarrollo Sostenible. Consejo Nacional para la Implementación de la Agenda 2030 de Desarrollo Sostenible.

Kozak, D. M. (2016). John FC Turner y el debate sobre la participación popular en la producción de hábitat en América Latina en la cultura arquitectónico-urbanística, 1961-1976. URBANA: Revista Eletrônica do Centro Interdisciplinar de Estudos sobre a Cidade, 8(3), 4968. https://doi.org/10.20396/urbana.v8i3.8646011

Lefebvre, H. (1975). El derecho a la ciudad. 3a ed. Península.

MacGuirk, J. (2015). Ciudades radicales. Un viaje a la nueva arquitectura latinoamericana. Turner Publicaciones.

Ministerio de Desarrollo Social y Familia. (2017). Ingresos de los hogares. Sintesis de Resultados CASEN 2017. http://observatorio.ministeriodesarrollosocial.gob. cl/storage/docs/casen/2017/Resultados ingresos Casen 2017.pdf

Ministerio de Desarrollo Social y Familia. (2018). Sintesis de resultados Encuesta Casen 2017: vivienda y entorno. http://observatorio.ministeriodesarrollosocial.gob. cl/storage/docs/casen/2017/Resultados vivienda casen 2017.pdf

Ministerio de Vivienda y Urbanismo. (s. f.). Catastro nacional de campamentos. https://www.minvu.cl/ catastro-de-campamentos/

Ministerio de Vivienda y Urbanismo. (2019). Informe metodológico Catastro Nacional de Campamentos 2019. Gobierno de Chile, MINVU.

Moreno, J. (2012). Estrategias de co-residencia de familias y hogares en Chile. Hacia un diagnóstico renovado en el contexto de los actuales desafíos de la política habitacional y urbana. V Congreso ALAP "Las transiciones en 
América Latina y el Caribe. Cambios demográficos y desafíos sociales presentes y futuros", Montevideo, Uruguay.

Moreno, L. (2016). La medición multidimensional de la pobreza en Chile como paso fundamental para la incorporación del enfoque de derechos en las políticas sociales. Revista Intervención, (6), 11-18.

Moser, C. 0. (1998). The asset vulnerability framework: reassessing urban poverty reduction strategies. World Development, 26(1), 1-19. https://doi.org/10.1016/ S0305-750X(97)10015-8

ONU. (1966). Pacto internacional de derechos económicos, sociales y culturales. https://www.ohchr.org/sp/professionalinterest/pages/cescr.aspx

ONU. (2015). Transformar nuestro mundo: la Agenda 2030 para el desarrollo sostenible._https://unctad.org/ system/files/official-document/ares70d1 es.pdf

ONU-Habitat. (1976). The Vancouver Declaration on Human Settlements. https://mirror.unhabitat.org/downloads/ docs/The Vancouver Declaration.pdf

ONU-Habitat. (2017). Nueva agenda urbana. Naciones Unidas. http://habitat3.org/wp-content/uploads/ NUA-Spanish.pdf

Ortiz, E. (1998). Notas sobre la producción social de la vivienda, elementos básicos para su conceptualización e impulso. Casa y Ciudad.

Ortiz, E. y Zárate, L. (2002). Vivitos y coleando. 40 años trabajando por el hábitat popular en América Latina. Universidad Autónoma Metropolitana- HIC-AL. Mexico.
Paquette Vassalli, C. (2020). Regeneración urbana: un panorama latinoamericano. Revista INVI, 35(100), 38-61. http://doi.org/10.4067/S0718-83582020000300038

Rivas, A. (2013). Campamentos: factores socioespaciales vinculados a su persistencia. (Tesis Magíster en Urbanismo, Universidad de Chile, Santiago). http://repositorio.uchile.cl/handle/2250/116946

Rodríguez, A. y Sugranyes, A. (2017). La nueva agenda urbana: pensamiento mágico. Hábitat y Sociedad, (10). https://doi.org/10.12795/HabitatySociedad.2017. $\underline{\mathrm{i} 10.10}$

Rodríguez, M. C., Di Virgilio, M. M., Procupez, V., Vio, M., Ostuni, F., Mendoza, M., y Morales, B. (2007). Producción social del hábitat y políticas en el Área Metropolitana de Buenos Aires: historia con desencuentros. Instituto de Investigaciones Gino Germani Facultad de Ciencias Sociales UBA. 


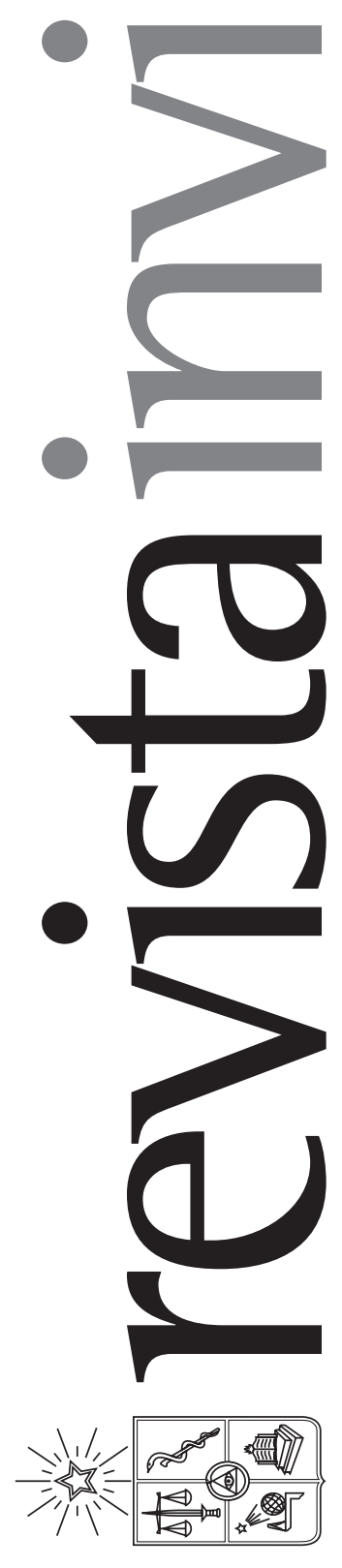

Revista INVI es una publicación periódica, editada por el Instituto de la Vivienda de la Facultad de Arquitectura y Urbanismo de la Universidad de Chile, creada en 1986 con el nombre de Boletín INVI. Es una revista académica con cobertura internacional que difunde los avances en el conocimiento sobre la vivienda, el hábitat residencial, los modos de vida y los estudios territoriales. Revista INVI publica contribuciones originales en español, inglés y portugués, privilegiando aquellas que proponen enfoques inter y multidisciplinares y que son resultado de investigaciones con financiamiento y patrocinio institucional. Se busca, con ello, contribuir al desarrollo del conocimiento científico sobre la vivienda, el hábitat y el territorio y aportar al debate público con publicaciones del más alto nivel académico.

Directora: Dra. Mariela Gaete Reyes, Universidad de Chile, Chile Editor: Dr. Luis Campos Medina, Universidad de Chile, Chile

Editores asociados: Dr. Gabriel Felmer, Universidad de Chile, Chile.

\section{Dr. Pablo Navarrete, Universidad de Chile, Chile.}

Dr. Juan Pablo Urrutia, Universidad de Chile, Chile

Coordinadora editorial: Sandra Rivera, Universidad de Chile, Chile.

Asistente editorial: Katia Venegas, Universidad de Chile, Chile.

\section{COMITÉ EDITORIAL:}

Dr. Victor Delgadillo, Universidad Autónoma de la Ciudad de México, México.

Dra. María Mercedes Di Virgilio, CONICET/ IIGG, Universidad de Buenos Aires, Argentina. Dra. Irene Molina, Uppsala Universitet, Suecia.

Dr. Gonzalo Lautaro Ojeda Ledesma, Universidad de Valparaíso, Chile.

Dra. Suzana Pasternak, Universidade de São Paulo, Brasil.

Dr. Javier Ruiz Sánchez, Universidad Politécnica de Madrid, España.

Dra. Elke Schlack Fuhrmann, Pontificia Universidad Católica de Chile, Chile.

Dr. Carlos Alberto Torres Tovar, Universidad Nacional de Colombia, Colombia.

Sitio web: http://www.revistainvi.uchile.cl/

Correo electrónico: revistainvi@uchilefau.cl

Licencia de este artículo: Creative Commons Atribución-Compartirlgual 4.0

Internacional (CC BY-SA 4.0) 